\title{
From particles to nanowires
}

\author{
Ideas that originated from particle and nuclear physics are now playing a prominent role in areas of \\ nanoscience and technology.
}

A considerable proportion of nanoscience research can, in some sense, be classified as condensed-matter physics, but concepts that originated from other, more estranged, branches of physics can also, on occasion, play a role. For

example, excitons in semiconductors, which are electronhole pairs bound by the Coulomb interaction, can be thought of as analogues of the hydrogen atom. Also, electrons in graphene can behave as if they have no mass and are best described using the relativistic Dirac equation rather than the Schrödinger equation.

In this issue, we highlight two such topics Majorana fermions and skyrmions.

Majorana fermions were first theoretically predicted in 1937 by the Sicilian particle physicist Ettore Majorana ${ }^{1}$ (pictured above). Through an analysis of the Dirac equation he proposed that some neutral fermions (the Majorana fermions) could be their own antiparticle. For comparison, elementary particles such as electrons or quarks have antiparticles - called positrons and antiquarks, respectively - with the opposite electric charge.

These elementary particles have yet to be detected (although it is still unclear whether neutrinos are in fact Majorana fermions). However, and as Marcel Franz explains in his Commentary in this issue ${ }^{2}$, it has recently been proposed that analogues of Majorana fermions could exist in solidstate nanostructures, emerging as chargeneutral, zero-energy quasiparticles known as Majorana zero modes. Interest in these states is primarily fundamental, driven by the possibility of finally studying an entity that was predicted over 70 years ago. However, it has also been suggested that they might be of use in quantum information processing.

A number of different research groups have recently reported experiments using semiconductor nanowires connected to superconductors, which reveal features in the electron transport characteristics that are consistent with the formation of Majorana zero modes. However, each observation has been indirect and could, in principle, be explained by phenomena unrelated to the existence of Majorana zero modes. As a result, direct and conclusive evidence for their existence remains elusive and, as Franz explains, is perhaps beyond our current experimental capabilities. There is, however, a growing body of theoretical and experimental evidence to support their existence, which could be further expanded by, for example, observing the anomalies in nanowires of topological insulators.

Twenty-five years after the predictions of Majorana, the British nuclear physicist Tony Skyrme (pictured below) proposed that protons and neutrons exist as topological solitons ${ }^{3}$ - that is, field configurations with particle-like character that are topologically protected from small distortions because their structure cannot be continuously deformed. The concept of these particles (or 'skyrmions') has since been applied to cosmology, particle physics and condensedmatter physics.

In condensed-matter physics, skyrmions can be found in magnetic materials where they exist as whirling spin textures with a well-defined nanoscale spatial arrangement. They were first observed in 2009 in bulk helical magnets ${ }^{4}$ and further experimental observations quickly followed. Skyrmion crystals have, for example, since been directly observed in magnetic thin films ${ }^{5}$ and at temperatures of up to $250 \mathrm{~K}$ (ref. 6).

Skyrmions have been proposed as information carriers in spintronic devices because of their intrinsic properties, such as their stability against structural distortions and the limited sensitivity of their motion to structural defects ${ }^{7,8}$. In a second Commentary in this issue ${ }^{9}$, Albert Fert, Vincent Cros and João Sampaio explore the potential, and challenges, of using these spin nanostructures in memory and logic devices.

The idea of using skyrmions as bits is similar to the idea of using magnetic domain walls - the boundaries between regions of opposite magnetization - as bits ${ }^{10}$. Here, electrical currents are used to transfer information encoded in the orientation of the magnetization of the wall or skyrmion. The advantage of skyrmions over domain walls is, however, the ease with which they can be moved by an electrical current. Skyrmions require current densities that are orders of magnitude lower than those needed for domain walls, which means much lower power consumption.

However, and as Fert and colleagues explain, before proof-of-concept devices can be made it will first be necessary to find ways to controllably generate single, or ensembles of, skyrmions in magnetic nanowires. Furthemore, real-world applications will require the discovery of materials in which skyrmions exist at and above room temperature.

The origins of Majorana fermions and skyrmions may lie in particle and nuclear physics, but the ideas are now beginning to leave their mark in nanoscience and technology. Given the pace and excitement of these fields, they will undoubtedly be the topic of numerous lively discussions at the upcoming March meeting of the American Physical Society, and we eagerly await the next major development. $\square$

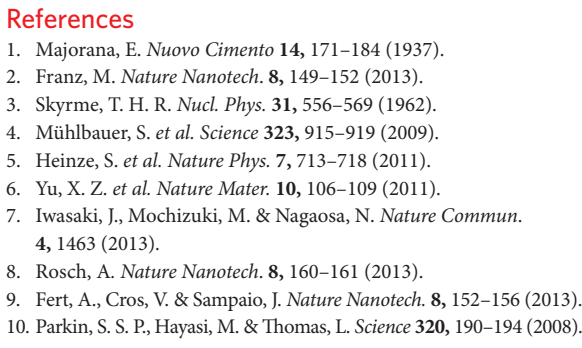




\section{Correction}

In the version of the Editorial 'From particles to nanowires' originally published (Nature Nanotechnology 8, 145; 2013), an image of the wrong Tony Skyrme was used; it should have been that shown below. This error has now been corrected in the HTML and PDF versions. Corrected after print 12 April 2013.

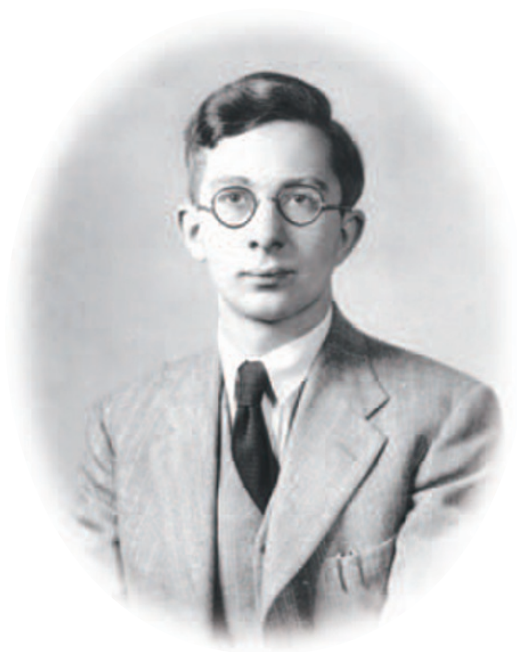

COURTESY OF THE MASTER AND FELLOWS OF

TRINITY COLLEGE CAMBRIDGE 